\title{
BAJO RENDIMIENTO ACADÉMICO : MÁS ALLÁ DE LOS FACTORES SOCIOPSICOPEDAGÓGICOS
}

\author{
María Estela Flores-Ortiz, Hilda Rivera-Coronel y Flavio Sánchez-Cancino \\ UNAM, FES IZTACALA \\ MÉXICO
}

\begin{abstract}
RESUMEN
Estudio que tuvo como objetivo conocer el grado de correlación entre el rendimiento académico y los factores sociopsicopedagógicos: historia escolar, proyecto de vida, hábitos y técnicas de estudio, relaciones con docentes, compañeros y familiares, para determinar si alguno de ellos estuviera relacionado directamente con el alto y bajo rendimiento. A 300 estudiantes de medicina se les aplicó el instrumento "Evaluación Integral del Rendimiento Académico a Nivel Superior" (Flores, Morales \& Landazuri, 2007) que contiene las subcategorías mencionadas anteriormente. Los resultados muestran que no se presentó una correlación directa (More, 2006), pues fluctuaron entre $r=.042$ a $r=.194$, del coeficiente de correlación de Spearman, donde fue empleado un nivel de significancia de .05 . Se concluye que los factores sociopsicopedagógicos estudiados son elementos que intervienen en el rendimiento académico, pero ninguno fue determinante; por tanto, en la interrelación que existe entre ellos no está la raíz del problema. Este estudio es una invitación para investigar si en los obstáculos definidos por Bachelard (1985) construidos en el transcurso de la historia inconsciente de cada estudiante pudiera estar la respuesta.
\end{abstract}

Palabras Clave:

Rendimiento académico, historia escolar, hábitos y técnicas de estudio, relaciones interpersonales, obstáculos epistemofílicos.

\section{LOW ACADEMIC PERFORMANCE: BEYOND SOCIOPSYCOPEDAGOGICAL FACTORS}

\begin{abstract}
Study aimed to determine the degree of correlation between academic performance and psycosocialpedagogic factors: school history, life plan, habits and study skills, relationships with teachers, classmates and relatives, to determine if any of them were directly related the high and low performance. 300 medical students were administered the instrument "Evaluación Integral del rendimiento Académico a Nivel Superior (Comprehensive Assessment of Academic Performance at Higher Level)" (Flores, Morales Sanchez, 2012) containing the subcategories mentioned in psycosocialpedagogic factors. The results show that a direct correlation (More, 2006) was submitted, as they fluctuated between 042-194, the Spearman's rank correlation coefficient, where it was used a significance level of .05, plus the difference between the number of answers given by the group of high (469) and low yield (550) was only $8 \%$. It is concluded that the psycosocialcialpedagogic factors studied are elements involved in academic performance but none was decisive, therefore the interrelationship between them is not the root of the problem, then what determines the way how the factors relate, so that the population of poor academic performance has been superior? This study is an invitation to investigate, if obstacles (Bachelard, 1985) built during the history of each student unconscious, could be the answer.
\end{abstract} Keywords:

Academic achievement, school history, habits and study skills, interpersonal relationships, epistemophilical obstacles

Bitácora del Artículo:

| Recibido: 4 de Agosto de 2015 | Aceptado: 4 de Septiembre de 2015 | Publicado en línea: Enero-Junio de 2016 | 


\title{
BAJO RENDIMIENTO ACADÉMICO : MÁS ALLÁ DE LOS FACTORES SOCIOPSICOPEDAGÓGICOS
}

\author{
María Estela Flores Ortiz, Hilda Rivera Coronel y Flavio Sánchez Cancino
}

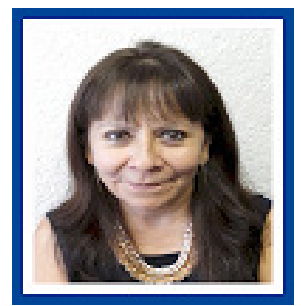

María Estela Flores Ortiz FESI, UNAM

Correo:

estela_fo@hotmail.com

Profesora de la Carrera de Psicología en el Área de Psicología Social Aplicada en la FES Iztacala, responsable del Programa Multidisciplinario de atención al Rendimiento Académico.

Ver más...

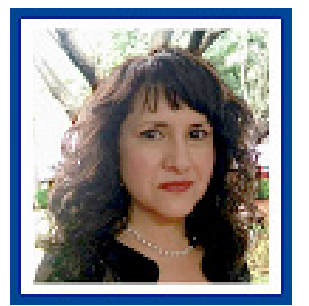

Hilda Rivera Coronel FESI, UNAM

Correo:

hilrivcor@yahoo.com

Profesora de la carrera de Psicología en el área de Psicología Social Aplicada en la FES Iztacala, maestra en tanatología y colaboradora del Programa Multidisciplinario de Atención al Rendimiento Académico.

Ver más...

\section{CONTRIBUCIÓN DE LAS AUTORAS}

Los autores María Estela Flores Ortiz, Hilda Rivera Coronel y Flavio Sánchez Cancino, participaron en la realización de todos los rubros, desde la concepción del estudio, el método, análisis de datos, conclusiones y discusión, así como en la revisión del manuscrito.

\section{AgRAdECIMIENTOS}

Agradecimiento especial a la carrera de Médico Cirujano de la FES Iztacala UNAM, por el apoyo otorgado para la realización de la presente investigación.

\section{Datos de Filiación de las Autoras}

FES Iztacala, UNAM| FES Iztacala, UNAM| FES Iztacala, UNAM

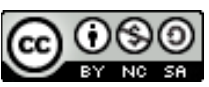

Copyright: (c) 2016 Flores-Ortiz. M.H., Rivera-Coronel, H. \& Sánchez-Cancino, F.

Este es un artículo de acceso abierto distribuido bajo los términos de la licencia Creative Commons Reconocimiento-NoComercial-Compartirlgual 4.0 Internacional, por lo que su contenido gráfico y escrito se puede compartir, copiar y redistribuir total o parcialmente sin necesidad de permiso expreso de sus autoras con la única condición de que no se puede usar con fines directamente comerciales y los términos legales de cualquier trabajo derivado deben ser los mismos que se expresan en la presente declaración. La única condición es que se cite la fuente con referencia a la Revista Digital Internacional de Psicología y Ciencia Social y a sus autoras. 


\section{TABLA DE CONTENIDO}

INTRODUCCIÓN

MÉTODO

Objetivo, 100

Hipótesis, 100

Tipo de Estudio, 100

Población, 100

Materiales, 100

Instrumento, 100

Procedimiento, 101

Mediciones, 101

Análisis estadísticos, 101

RESULTADOS

CONCLUSIONES 


\section{INTRODUCCIÓN}

E momento que vive la educación en México es preocupante. Avilés (2007) señala que desafortunadamente 12 millones de niños mexicanos no asisten a la escuela y que de cada 100 que inician su formación básica 78 la concluyen, en secundaria 68 , en preparatoria 24 y únicamente 14 terminan una licenciatura, implicando una pérdida del $86 \%$ para el sistema educativo y el país.

Para el año 2015 los resultados no son más alentadores; Rodríguez, Jiménez y Beltrán (2015) señalan que aproximadamente el $25 \%$ de cada 100 estudiantes a nivel superior abandonan su formación en el primer semestre. La mayoría de ellos inician con materias reprobadas y bajos promedios, en el tercer semestre se presenta una deserción del 36\%, cifra que se incrementa semestre a semestre, hasta alcanzar el $46 \%$.

Aunado a lo anterior y según los últimos resultados obtenidos por "El Programme for Internacional Student assessment 2012", hoy mejor conocido como la evaluación de PISA, los adolescentes mexicanos de quince años que estudian, se encuentran en los últimos lugares de los 60 países participantes, en ciencia se encuentran en el lugar 55, seguido por matemáticas en el 53 y lectura en el 52. Si bien es cierto que hay un avance de 28 puntos en matemáticas, en los resultados del 2003, la realidad es que México continúa por debajo del promedio obtenido por los países pertenecientes a la Organización para la Cooperación y el Desarrollo Económicos (OCDE), lo que significa que los estudiantes mexicanos tienen un retraso en las competencias de al menos dos años, de tal forma que alcanzar los niveles promedio actuales en matemáticas tardarán 25 años y 65 en lectura (OCDE, 2012).

Las cifras anteriores permiten que Izquierdo y Ulloa (como se citó en Flores \& Sánchez, 2012), consideren que los jóvenes mexicanos tienen el mínimo de las competencias necesarias para desempeñarse con solvencia en las sociedades contemporáneas; presentan graves deficiencias para iniciar sus estudios profesionales y por supuesto tendrán graves dificultades para insertarse con éxito tanto al mercado laboral como a los grupos sociales, científicos, políticos y empresariales que dirigen al país.

Los datos obtenidos por la OCDE son preocupantes, y lo son más cuando los estudiantes al ingresar a la universidad son dependientes, inseguros, sin resistencia a la frustración, con dificultades para organizar su tiempo, con hábitos de estudio deficientes, el tiempo que dedican a estudiar es en promedio dos horas algunos días de la semana, sus decisiones están mayormente centradas en aspectos lúdicos, e ingresan a la universidad con la idea de que realizar estudios profesionales no requiere de gran esfuerzo (Flores, Morales, Sánchez, 2004; Flores, O., Guarneros, R., Sánchez, C. \& Silva, R., 2009).

Las características anteriores dificultan que las instituciones de educación superior logren formar profesionales altamente capacitados para enfrentar las demandas sociales y así garantizar su ingreso al mundo laboral. En el proceso se presentan dificultades en la aprehensión de conocimientos que se manifiestan en bajas calificaciones, reprobación de materias, deserción, tiempo extra de estancia en la universidad y dificultad para concluir los requisitos para la obtención del título; todos estos problemas se pueden englobar en un concepto denominado bajo rendimiento académico.

Diversas son las estrategias que las instituciones educativas emplean para subsanar las problemáticas antes planteadas, pero es un hecho que generalmente se centran en resolver el problema de las calificaciones a través de exámenes extraordinarios, asesorías, tutorías, apoyos en línea, grupos de recursamiento, entre los más relevantes; atacando así el síntoma más no el origen, y por esta razón la problemática se resuelve momentáneamente, pero las causas que generan los conflictos permanecen, convirtiéndose el problema en un proceso cíclico.

Asimismo, las universidades se empeñan en la actualización de sus planes y programas de estudio con la finalidad de que sus contenidos correspondan a las necesidades sociales actuales; desafortunadamente, aunque estos esfuerzos son importantes, la modificación o el cambio de un plan de estudio no erradica la problemática escolar, más bien plantea nuevos retos (Garbanzo, 2007).

Lo antes expuesto plantea que mientras no se enfrenten las causas que generan el bajo rendimiento académico, es muy difícil que las universidades cumplan su objetivo central "formar profesionistas de calidad a nivel mundial" $y$ por lo tanto ubicarse entre las mejores del mundo.

\section{El Rendimiento Académico y Su Complejidad}

La complejidad al abordar el tema del rendimiento académico y sus problemáticas inicia desde la percepción ambivalente que socialmente se tiene, por una parte se considera que es un elemento central en el proceso de aprendizaje, pero la cotidianidad en que se desarrolla produce que se vea como algo natural, restándole importancia y significado.

Dilucidar los elementos que están presentes en este proceso y diferenciar aquellos que derivan en un alto o 
bajo rendimiento académico ha sido el objeto de estudio de disciplinas como la pedagogía, la psicología y en menor grado la medicina. Sin embargo, la complejidad del problema y la diversidad de los enfoques de quienes lo estudian han derivado en investigaciones aisladas que en su mayoría no presentan estrategias de intervención. Lo anterior es muy sencillo observarlo cuando a esta problemática se le denomina con términos como: aprovechamiento escolar, rendimiento escolar y rendimiento académico como sinónimos, pero cada autor lo define según su perspectiva. Por lo tanto, una definición generalizada no existe y tampoco un acuerdo de que signifiquen lo mismo.

Flores y Sánchez (2014) mencionan que el proceso de estudio del rendimiento académico ha transitado en tres momentos que a continuación se presentan:

\section{Primero: El alumno como el único responsable}

Jiménez (como se citó en Edel, 2003) define como rendimiento escolar el nivel de conocimientos demostrado por el alumno en un área o materia comparado con la norma de edad y nivel académico; Castro (2007) considera que es el grado alcanzado por los estudiantes, o bien como la capacidad del alumno para el logro de un objetivo (Basadre, 2008).

Esta forma de mirar el problema propició que los investigadores se interesaran por el estudio de factores propios del estudiante en el área biológica (salud, alimentación y ejercicio) y en la psicológica (personalidad, inteligencia, motivación, autoconcepto, perseverancia, etcétera). A continuación se citan algunos ejemplos de las investigaciones realizadas.

Resultados obtenidos tanto en la Encuesta Nacional de Salud en Escolares (ENSE) realizada en el 2008 a nivel básico, como la investigación de Ruvalcaba et al. (2012) a estudiantes universitarios, coinciden que la ingesta exagerada de los Ilamados alimentos chatarra con alto contenido de calorías, colorantes y saborizantes artificiales, así como un bajo consumo de los nutrientes necesarios para el funcionamiento del organismo, repercute negativamente en el grado de energía, produce irritabilidad, falta de concentración; además, el sedentarismo y la alta ingesta de alimentos generan obesidad que repercute en el autoconcepto y autoestima, tan importantes en el aprendizaje.

Por su parte Caldera \& Pulido (2007) identificaron la relación que existe entre el estrés y el rendimiento académico en estudiantes de psicología, encontrando que no hubo una relación significativa, dato que coincide con Flores, Guarneros, Sánchez y Silva en el 2009, con estudiantes de la carrera de medicina.
Por otra parte, Almeida, Guisande, Primi y Lemos (2008) establecieron una relación entre los test de inteligencia y las calificaciones escolares, concluyendo que a medida que se avanza en la escolarización hay una menor correlación entre inteligencia y rendimiento escolar. Asimismo, Garbanzo (2007) y Artunduaga (2008) encontraron que en la medida que el estudiante se considera autocompetente y con un elevado autoconcepto, su desempeño académico se ve favorecido. Con base en una correlación directa y positiva, Gómez, Oviedo y Martínez (2011) concluyeron que el interés por la carrera que estudian es un factor favorable para su rendimiento académico. Por su parte, Izar, Ynzunza y López (2011) mencionan que existe una relación directa entre el rendimiento académico y aquellos que tienen un proyecto de vida bien definido y una historia escolar favorable. Por su parte Bartual y Poblet (2009) encontraron que la asistencia periódica a clase y el trabajo constante son factores que incrementan el porcentaje de éxito de los alumnos.

Los estudios presentados son una muestra de la gran diversidad de factores que los investigadores han abordado para definir la relación que tienen con el rendimiento académico y su problemática. Sin embargo, a pesar de la importancia de sus resultados, todos ellos aclaran que son elementos que lo contribuyen pero que no son determinantes y mucho menos se pueden generalizar; por tanto, sería imposible afirmar que un solo factor impide el éxito en el rendimiento académico, por ejemplo, si todos los alumnos tuvieran un autoconcepto alto, ello ¿garantizaría el éxito académico?

\section{Segundo: El alumno y otros factores y actores}

La visión de otros investigadores fue más allá de lo individual, se interesaron por analizar la intervención de otros actores como docentes, compañeros, padres de familia y autoridades, o bien factores como programas, contenido de las materias, situación económica, etcétera (Correa, 2004; Vilchis, 2006; Vittet, 2008). Asimismo, la composición familiar y su dinámica definen el apoyo que brinda la familia al estudiante. Torres y Rodríguez (2006) encontraron que existe una relación positiva entre el apoyo familiar y el éxito escolar en estudiantes universitarios.

También el ser mirados como exitosos o fracasados por profesores y padres de familia, influye de una manera importante en su desempeño académico, incluso Díaz (2000) va más allá al considerar que el estilo de crianza durante los primeros años de vida son fundamentales, pues en ellos se originan los mecanismos intelectuales, los medios lingüísticos y actitudes para la solución de tareas, en donde las familias alejadas de la 
cultura del saber propician una actitud de menor éxito académico entre sus miembros.

En relación a los factores que se presentan en el proceso educativo, desde hace al menos 30 años Juárez (1985), también investigó las formas de evaluación, pues es el mecanismo que regula y establece los estándares para ser considerados como los más aptos en los conocimientos y habilidades de la época, según lo requerido por el momento que vive la sociedad.

Las investigaciones anteriores son una muestra del interés que se ha tenido desde muchos años atrás, para demostrar que el alumno no es el único responsable, pero también es importante remarcar que son estudios que establecen una relación de uno a uno, es decir el alumno y uno de tantos factores.

\section{Tercero: Como resultado de un proceso}

Una tendencia reciente considera al rendimiento académico como el resultado de un proceso complejo, donde intervienen actores como son alumnos, padres de familia, docentes, compañeros y directivos, así como los factores biológicos, psicopedagógicos, sociales y ambientales (Margot, 2006; Pizarro, 2007; Flores, et al., 2007, 2009, 2014; Vásquez, C., Cavallo, M., Aparicio, M., Muñoz, B., noviembre 2012)

Lo antes mencionado permite concluir que el bajo rendimiento académico, como problemática compleja, ha sido una preocupación constante de alumnos, docentes, padres de familia, directivos y por supuesto de diversos investigadores; sin embargo, la visión lineal que ha predominado en la forma de ver e intervenir en el objeto de estudio ha sido insuficiente para entender y solucionar el problema. Por tanto, se torna necesario estudiar al rendimiento académico desde un enfoque multifactorial, con la finalidad de conocer el grado de interrelación que existe entre factores y actores en el bajo rendimiento académico.

\section{Método}

\section{Objetivo}

El objetivo del presente estudio es conocer el grado de correlación que existe entre el rendimiento académico y los actores: alumnos, profesores, padres de familia, así como con los factores sociopsicopedagógicos (historia escolar, proyecto de vida, hábitos y técnicas de estudio) y su impacto en la problemática escolar.

\section{Hipótesis}

Existe una amplia diferencia en la correlación de los factores sociopsicopedagógicos y el rendimiento académico entre ambos grupos.

\section{Tipo de Estudio}

Estudio de tipo transversal, cuantitativo y correlacional de Spearman, en el que fue empleado un nivel de significancia de .05, donde se estableció la relación entre el bajo rendimiento académico y los factores sociopsicopedagógicos: historia escolar, proyecto de vida, hábitos y técnicas de estudio, relaciones con docentes, compañeros y familiares.

\section{Población}

La muestra se seleccionó por conveniencia, definida como una técnica no probabilística, donde la población es seleccionada desde la accesibilidad y proximidad de los sujetos para el investigador (Creswell \& Garrett, 2008).

Participaron 300 estudiantes de nuevo ingreso a la carrera de Médico Cirujano de la Facultad de Estudios Superiores, Campus Iztacala UNAM, de ambos sexos, cuyas edades fluctuaron entre los 18 y 20 años.

\section{Materiales}

300 formatos de la "Evaluación Integral del Rendimiento Académico para el Nivel Superior (Flores et al., 2007) y lápices.

\section{Instrumento}

Se aplicó el instrumento denominado "Evaluación Integral del Rendimiento Académico para el Nivel Superior" (Flores et al., 2007) que consta de cinco unidades de análisis: biológica, psicológica, pedagógica, social y ambiental, a través de 112 preguntas cerradas y abiertas.

\section{Procedimiento}

La aplicación del instrumento se realizó en grupos de 30 estudiantes cada uno, por un espacio de dos horas, en los salones de la Facultad.

\section{Mediciones}

\section{Unidades de análisis:}
A. RENDIMIENTO ACADÉMICO
1. Alto: estudiantes con un promedio de 8 o mayor.
2. Bajo: estudiantes con un promedio menor a 8 . 


\section{B. FACTORES PSICOLÓGICOS}

1. Historia escolar favorable: que los alumnos no hubieran reprobado ningún ciclo

escolar y que sus calificaciones fueran mayores de 7.

2. Proyecto de vida definido: tener metas a corto y mediano plazo tanto en su formación profesional como en su vida.

\section{FACTORES PEDAGÓGICOS}

1. Hábitos y técnicas de estudio favorables: tener un espacio destinado para estudiar, horarios y tiempos definidos, así como al menos dos técnicas de

estudio que las empleen cotidianamente.

D. FACTORES SOCIALES: RELACIONES

1. Alumno-profesor: vínculo de comunicación, respeto y confianza que favorezca el aprendizaje.

2. Alumno-compañeros: vínculo de comunicación, respeto y confianza que favorezca el aprendizaje.

3. Alumno-familiares: vínculo de amor, comunicación, respeto, apoyo y confianza.

\section{Análisis estadísticos}

Para el análisis de los resultados se utilizó el coeficiente de correlación de Spearman, el cual permite obtener una correlación lineal entre dos variables cuantitativas (Durkemhein, 2005), a través de una escala ordinal que oscila entre -1 y +1 , indicando asociaciones negativas o positivas respectivamente, en donde 0 significa ausencia de correlación (More, 2006).

\section{Resultados}

Los resultados muestran que no se presentó una correlación directa (More, 2006), pues estos fluctuaron entre .042 a .194, del coeficiente de correlación de Spearman, donde fue empleado un nivel de significancia de .05. Como se puede observar en la tabla 1, en la columna " $A$ ", se presenta la población que contestó cada subcategoría; es interesante observar como ninguna fue contestada completamente por los 300 estudiantes. Sin embargo, historia escolar, hábitos y técnicas de estudio, y relaciones con los compañeros fueron las que los estudiantes contestaron más, contrario a las relaciones con los profesores que sólo lo hicieron 183.

La columna " $\mathrm{C}$ " representa al universo de los estudiantes incluidos en la categoría de favorable; en ella se observa que proyecto de vida obtuvo la mayor puntuación con 250 estudiantes, seguida por historia escolar (228) y relaciones con compañeros (220). Mención especial tiene la subcategoría relaciones favorables con los profesores que obtuvo la respuesta de sólo 3 alumnos.
En las columnas " $\mathrm{D}$ " $\mathrm{y}$ " $\mathrm{E}$ " se aprecian las cifras obtenidas por los alumnos de alto y bajo rendimiento, en donde es claro observar que, aunque las frecuencias son muy similares, las que presentan el bajo rendimiento son más altas.

Como resultado de lo anterior, en la columna " $\mathrm{F}$ " se presenta el índice de correlación que, según las cifras obtenidas, se aprecia que la correlación más alta se ubicó en la relación con los compañeros (.194), seguida por el proyecto de vida definido con .179 e historia escolar favorable con .174; los índices de correlación más bajos fueron para la relación favorable profesor alumno con .071, hábitos y técnicas de estudio .049, y relaciones familiares favorables .042.

\section{Tabla 1.}

Concentrado de los resultados obtenidos en las subcategorías

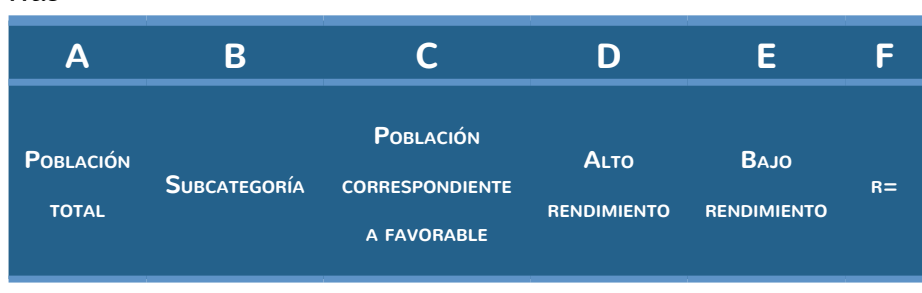

Historia

escolar favorable

228

107

121

.174

250

Proyecto de vida definido

250

114

136

.179

264

Hábitos y técnicas de estudio favorables

261

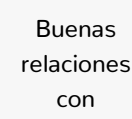
compañeros

231

Buenas relaciones con la familia 176 220 143 66 77 .049

Relaciones favorables con sus profesores
3

2

.071 
En la figura 1 se observa, a nivel de porcentajes, que en todas las subcategorías predominaron las respuestas dadas por los alumnos de bajo rendimiento, con excepción de la correspondiente a la de relaciones con los profesores.

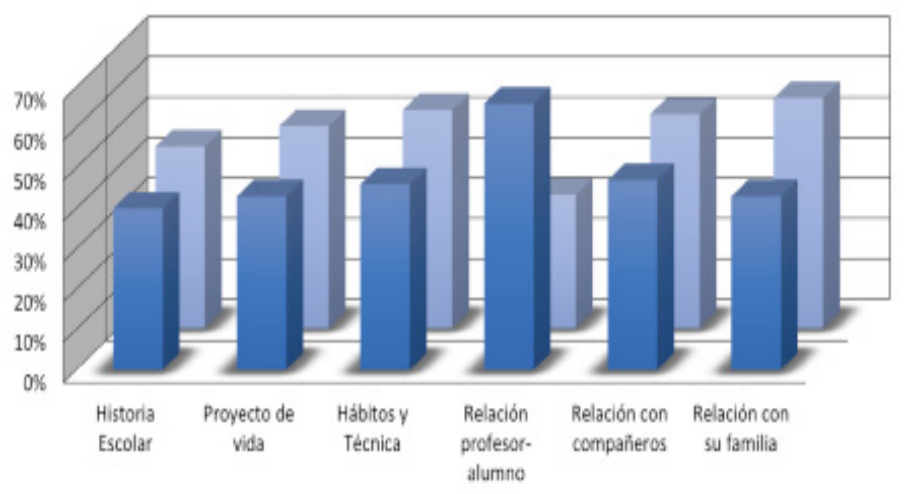

Alto rendimiento Bajo rendimiento

Figura 1.

Porcentajes de la relación de factores con alto y bajo rendimiento

La figura 2 es una representación de la proporción de cada subcategoría en el rendimiento académico, a pesar de que ninguna de ellas tuvo una correlación positiva alta; historia escolar, proyecto de vida y relaciones con los compañeros fueron las de mayor peso.

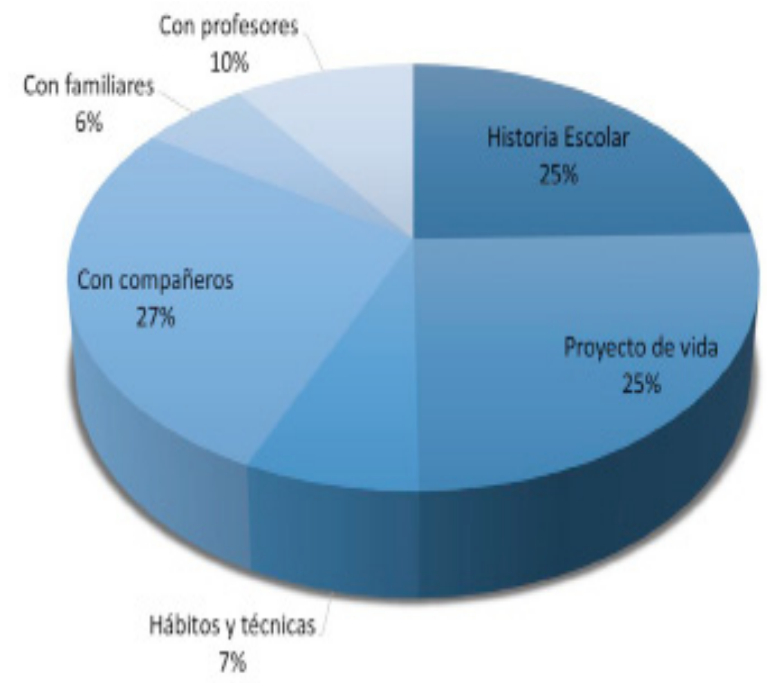

Figura 2.

Proporción de la relación de las subcategorías en el rendimiento académico

\section{CONCLUSIONES}

El que un estudiante obtenga bajas calificaciones, repruebe materias o algún año escolar, socialmente tiene un doble significado. Por un lado, se le señala, se le juzga y se le estigmatiza porque no es brillante, inteligente y con ganas de tener éxito, pero por otro, se justifica considerando que es algo normal y que sucede en cada familia y grado escolar, desde el jardín de niños hasta estudios de posgrado y que no hay porque alarmarse. ¿Realmente es normal? ¿No hay por qué preocuparse? ¿Sólo el tiempo lo solucionará? Son algunas de las preguntas que diversos especialistas se hicieron para transformar lo cotidiano en un problema a investigar.

El punto de partida, como era de esperarse, centró el problema en el alumno y por lo tanto las investigaciones giraron en torno a su salud, inteligencia, autoestima, autoconcepto y poco a poco se fueron enfocando a factores muy específicos como la frecuencia con que asiste a clases, la motivación que tiene sobre la carrera que estudia, etcétera.

Las reflexiones de las investigaciones realizadas, teniendo como punto de partida al alumno como el sujeto de estudio, dieron pauta a preguntarse ¿los profesores contribuirán para que algunos estudiantes tengan deficiencias en su aprendizaje?, ¿y las condiciones familiares? y ¿los programas y contenidos? Cuestionamientos que en el transcurso del tiempo se estudiaron y demostraron su participación en el rendimiento académico, pero nuevamente sólo afectaron una parte del problema.

Es innegable la aportación que los diversos investigadores han realizado en el transcurso de la historia; sin embargo, ambas visiones están fragmentadas porque se centraron en la desintegración y visión parcial del objeto a construir, indagando sobre el impacto que tiene un solo factor en la problemática escolar. Entonces la visión que hasta el momento predominaba era que la solución de la problemática podría estar centrada en encontrar un factor determinante, intervenir en él y con ello solucionar el problema.

Estudios recientes han dejado esta postura para considerar al bajo rendimiento académico como el resultado de un proceso, donde la interrelación de factores y actores determinan al bajo rendimiento académico. En este sentido, los resultados de esta investigación muestran, al igual que Corengia (2005) y Vargas (2012), que los factores investigados tienen relación con la problemática, aunque ninguno de ellos tiene el peso necesario para garantizar que en su intervención esté la solución del problema.

Izar, Ynzunza y López (2011) mencionan que los alumnos con una historia escolar favorable tendrán mejores oportunidades en el aprendizaje; sin embargo, los resultados obtenidos muestran lo contrario, porque si 
las subcategorías correspondían al grupo denominado como "favorables", se esperaba que predominaran los alumnos de alto rendimiento académico y no fue así. Asimismo, y de acuerdo con Artunduaga (2008), las relaciones con los profesores son importantes para generar un clima escolar favorable, por tanto, si sólo 3 de los 300 alumnos tienen buenas relaciones con sus profesores se considera necesario profundizar en este aspecto, debido a que ha sido un factor poco estudiando (Vásquez, et al., 2012); aunque es claro que sólo será una contribución más y no la solución del problema.

Por lo tanto, se concluye que:

1. Existe una correlación proporcional entre los factores estudiados y el rendimiento académico.

2. Por tanto, ninguno de los factores fue determinante para el proceso de aprendizaje.

3. El hecho de que tengan factores favorables, no garantiza un alto rendimiento.

3. Llama la atención que, de 300 estudiantes, sólo 3 hayan considerado que tienen buenas relaciones con sus profesores y que dos de ellos tengan alto rendimiento.

4. Tradicionalmente se piensa que aprender y aplicar técnicas de estudio solucionará el problema, pero los resultados muestran que es insuficiente.

5. Por tanto se considera que el rendimiento académico es el resultado de la interrelación de diversos factores y actores, pero ahora se presenta una nueva problemática, si ambas poblaciones (alto y bajo rendimiento) obtuvieron correlaciones similares, ¿qué determina que un estudiante tome un camino u otro?

Entonces se considera que las raíces del problema van más allá de la simple interrelación de factores y actores (nivel objetivo), falta un elemento que determine el sentido y el significado que cada estudiante dará a cada uno de ellos.

La historia del sujeto-educativo inició en el mismo instante en que fue deseado o no por sus padres, y en el transcurso de los años el discurso, acciones y sentimientos de sus personajes centrales lo fueron constituyendo como sujeto, y en este proceso las experiencias vividas las definió como buenas o malas, incrustándose como las herramientas con las cuales opera en el presente, algunas como elementos positivos con las cuales enfrentará la realidad, pero los negativos se trasformarán en obstáculos que dificultarán su vida cotidiana.

Por lo antes expuesto nos preguntamos: ¿Las bajas correlaciones que se encontraron en este estudio, representan tan sólo los factores presentes en el rendimiento académico?, pero no será ¿qué lo que determina al bajo rendimiento académico son los obstáculos que los estudiantes formaron en su experiencia de vida?, y por lo tanto ¿determinarán la manera de enfrentar las problemáticas en su proceso de enseñanza-aprendizaje y generará las bajas calificaciones? Entonces, ¿la falta de motivación, la flojera, la dificultad de los contenidos, etcétera, tan sólo serán la manifestación de los obstáculos y no la génesis?

Finalmente pensar el bajo rendimiento académico como el producto de la interrelación entre actores y factores, articulados por la historia inconsciente del sujeto en un momento determinado, constituye otra visión en la manera de analizar el problema, donde se traspase la visión de que el alumno "no quiere", "no le interesa", "es flojo", a que no puede hacerlo porque tiene obstáculos denominados por Bachelard (1985) como epistemofílicos, los cuales deberán develarse para solucionarlos y así favorecer el aprendizaje. Sólo de esta manera se podrá entender el verdadero proceso de formación, donde se vincule el pensar, el sentir y el actuar para su transformación y de la realidad (Pichon-Rivière, 1990).

\section{Referencias}

Almeida, L. S., Guisande, M. A., Primi, R. \& Lemos, G. (2008) Contribuciones del factor general y de los factores específicos en la relación entre inteligencia y rendimiento escolar. European Journal of Education and Psychology, 1(3), 5-16. Recuperado de htpp://www.redalyc.org/articulo.oa?id=129311870001

Artunduaga Murillo, M. (2008). Variables que influyen en el rendimiento académico en la universidad. Departamento MIDE (Métodos de investigación y diagnóstico en educación), Universidad Complutense de Madrid (España).

Avilés, K. (15, octubre 2007). Sólo el 14\% de los estudiantes llegan a la universidad. La Jornada Recuperado de http://www.jornada.unam.mx/2007/10/15/index. php?section $=$ sociedad $\&$ article $=044 \mathrm{n} 1 \mathrm{sc}$

Bachelard, G. (1985). La formación del espíritu científico. México: Siglo XXI Editores

Bartual, T. \& Poblet, M. C. (2009). Determinantes del rendimiento académico en estudiantes universitarios de primer año de Economía. Revista de Formación e Innovación Educativa Universitaria, 2(3), 172-181. Recuperado de http://refiedu.webs.uvigo.es/Refiedu/Vol2 3/ REFIEDU 2_36.pdf

Basadre, J. (2008). Educa a los Niños para No Castigar a los Adultos. SanDiego: Ed. Mullin.

Caldera M., \& Pulido C. (2007). Niveles de estrés y rendimiento académico en estudiantes de la carrera de Psicología del Centro Universitario de Los Altos. Recuperado de http://repositorio.cualtos.udg.mx:8080/jspui/ handle/123456789/319

Castro, P. (2007). El Fracaso Escolar. Madrid: Herder.

Creswell, J. W., \& Garrett, A. L. (2008). The" movement" of mixed methods research and the role of educators. South African Journal of Education, 28(3), 321-333.

Corengia, P. A. (2005). Rendimiento Académico en la Universidad. Disponible en:

http://web. austral. edu. ar/descargas/institucional/08. pdf (Recuperado en agosto 15 de 2012)

Correa, J. (2004). Una Visión Multidisciplinaria para el Rendimiento Académico. Venezuela: Alloy. 
Díaz, B. (2000). El examen. Textos para su historia y debate. México: Centro de Estudios sobre la Universidad y Plaza y Valdés Editores.

Durkemheim, P. (2005). Análisis Estadístico con SPSS para Windows. Panamá: Johnson.

Edel Navarro, R. (2003). El rendimiento académico: concepto, investigación y desarrollo. REICE: Revista Electrónica Iberoamericana sobre Calidad, Eficacia y Cambio en Educación.

Flores, O., Morales, R. \& Sánchez, C. (2004). Rendimiento académico: problemas y soluciones. Conferencia en el Simposio de Investigación FESI-UNAM.

Flores, O., Morales, R. \& Landázuri, M. (2007). Rescatemos a los niños. Ciudad de México: Grupo Editorial Éxodo.

Flores, O., Guarneros, R., Sánchez, C. \& Silva, R. (2009). El perfil real de los alumnos de nuevo ingreso de la carrera de médico cirujano: el punto de partida para la formación profesional. Revista Psicología y Ciencia Social, 11, 24-31.

Flores. O. \& Sánchez, C. (2012). Cómo Evaluar al Rendimiento Académico. México: Grupo Editorial Éxodo.

Flores, O. \& Sánchez, S. (2014). El taller: un dispositivo grupal para afrontar los obstáculos presentes en el rendimiento académico. Revista de Psicología y Ciencias Social, 14 (2).

Garbanzo, G. (2007). Factores asociados al rendimiento académico en estudiantes universitarios, una reflexión desde la calidad de la educación superior pública. Revista Educación, 31(1), 43-63.

Gómez, Oviedo \& Martínez. (2011). Factores que influyen en el rendimiento académico del estudiante universitario. Revista Tecnociencia Chihuahua, 5(2).

Izar, J. M., Ynzunza, C, B. \& López, H. (2011). Factores que afectan el desempeño académico de los estudiantes de nivel superior en Rioverde, San Luis Potosí, México. CPU-e, Revista de Investigación Educativa, 12. Recuperado de http://www.uv.mx/epue/num12/opinion/lzardesempeñoacademico.html.

Juárez, F. (1985). La prueba de opción múltiple y su validación académica. En Díaz, B. A. (2000). El examen. Textos para su historia y debate. México: Plaza y Valdés.

Margot, T. (2006). El Rendimiento Académico: Un Mundo que Envuelve al Estudiante. México: Ed. Agnati.

More, T. (2006). Análisis de Datos en Psicología más Estadística. Bogotá: Ed. Luna.
OCDE, (2012). Programa para la Evaluación Internacional de Alumnos PISA Resultados. Recuperado de http://www.oecd.org/pisa/keyfindings/PISA-2012-resultsmexico-ESP.pdf

Páez, C. M. \& Castaño C. J. (2014). Perfil personal, familiar y social del estudiante de medicina de la universidad de Manizales. Archivo de Medicina (Col.) Sin mes, 18-30.

Pizarro, K. (2007). La Revolución del Pensamiento en el Tercer Milenio. Argentina: Narcea.

Pichon-Rivière, E. (1990). Del psicoanálisis a la psicología social (Vol. 1). Buenos Aires: Nueva Visión.

Rodríguez, N. J., Jiménez, D. C. \& Beltrán, S. B. (2015). La educación superior en México, una demanda con compromiso social. RIDE Revista Iberoamericana para la Investigación y el Desarrollo Docente, 5 (10).

Ruvalcaba, C., Sánchez, C., Montes, M., Durán, V., Ortigosa, J. \& Morales, R. (2012). La mirada unidisciplinaria. En cómo Evaluar al Rendimiento Académico. México: Grupo Editorial Éxodo.

Torres, L. \& Rodríguez, S. (2006). Rendimiento académico y contexto familiar en estudiantes universitarios. Revista Enseñanza e investigación en psicología, 11(2), 255-270. Universidad Veracruzana.

Vargas, G. (2012). Factores asociados al rendimiento académico en estudiantes universitarios, una reflexión desde la calidad de la educación superior pública. Revista Educación, 31(1), 43-63.

Vásquez, C., Cavallo, M., Aparicio, M., Muñoz, B. (noviembre, 2012). Factores de impacto en el rendimiento académico universitario un estudio a partir de las percepciones de los estudiantes. Decimoséptimas Jornadas "Investigaciones en la Facultad de Ciencias Económicas y Estadística" Instituto de Investigaciones. Teóricas y Aplicadas. Escuela de Contabilidad. Universidad Nacional de Rosario.

Vilchis, M. (2006). Las Relaciones Interpersonales en la Escuela. México: Ed. Oxígeno.

Vittet, M. (2008). Condiciones para el bajo rendimiento académico. México: Belijan. 
Revista Digital Internacional de Psicología y Ciencia Social |Vol. 2 | Núm. $1 \mid$ Enero-Junio 2016 | ISSSN 1405-5802

\section{Meta-Análisis del Artículo}

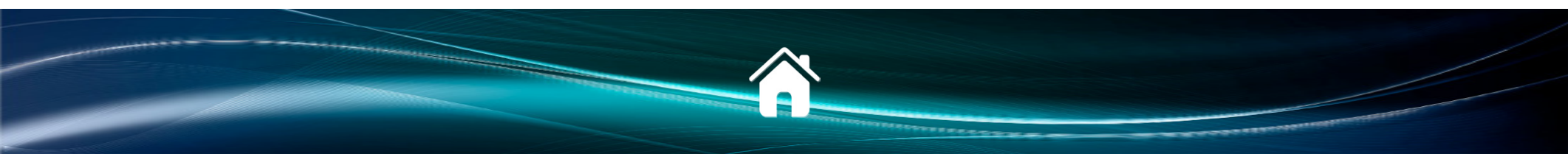


Artículo Empirírico | Bajo rendimiento académico: más allá de los factores...I Flores-Ortiz, Rivera-Coronel ez Sánchez-Cancino

\section{Dimensión Cuantitativa}

\section{Perfil de Evaluación entre pares}
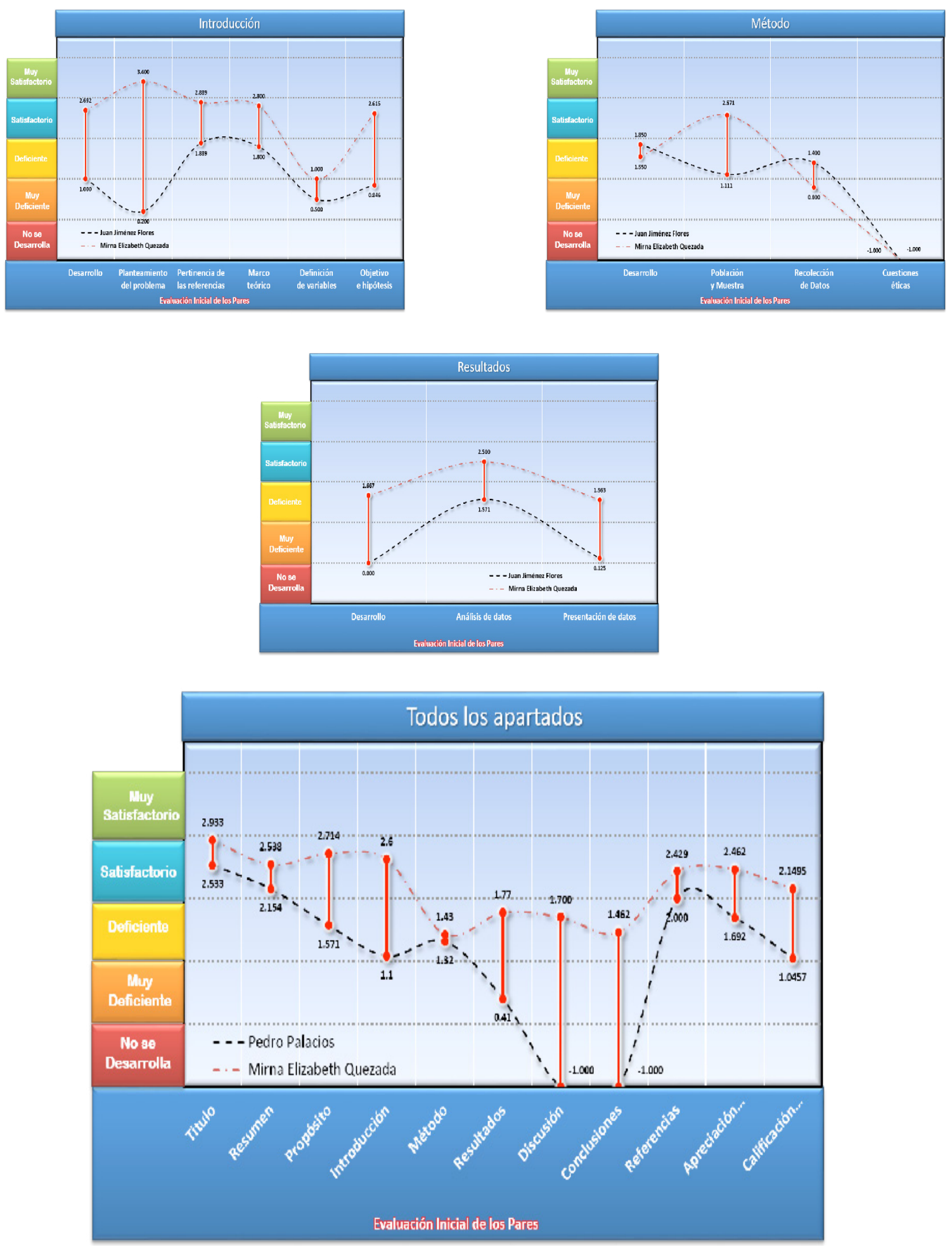


\section{Índice de Concordancia}

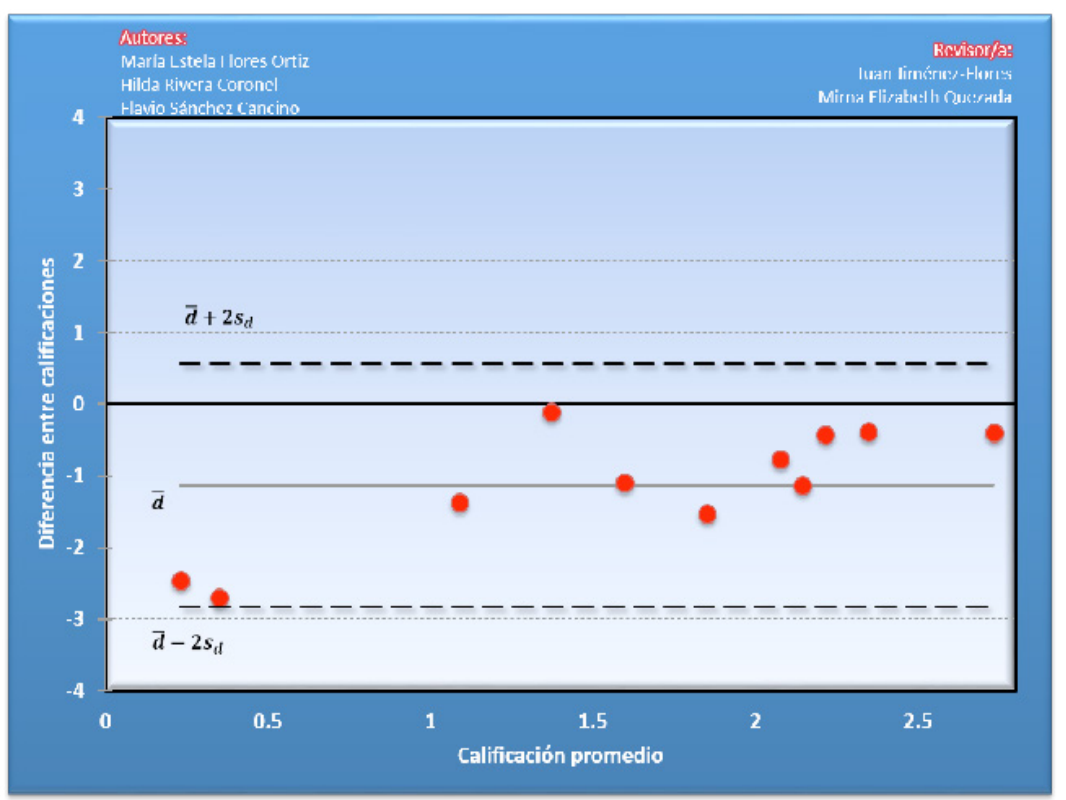

Índice de Acuerdo
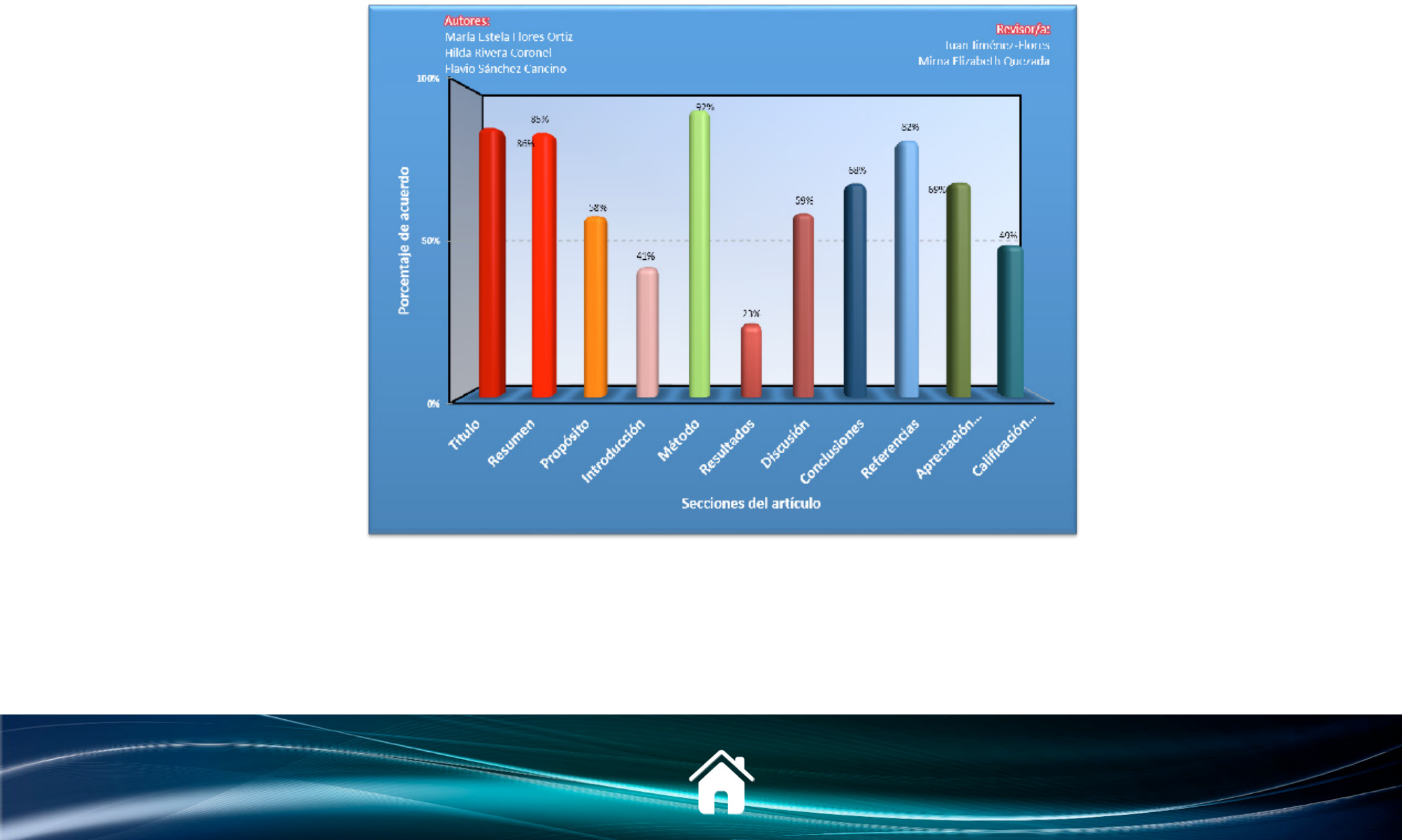


\begin{tabular}{|c|c|}
\hline Revisor 1 & Revisor 2 \\
\hline Mirna Elizabeth Quezada & Juan Jiménez-Flores \\
\hline
\end{tabular}

\section{Título/Autoría}

El título se puede mejorar. Me queda claro que en este artículo se revisará lo referente al bajo rendimiento académico, pero el resto del título no menciona de qué factores se están hablando. Podrían ser factores familiares, sociales, psicológicos, económicos, etc. Por lo tanto, para ser más específicos Mencionar los factores a los que se refiere el estudio. El título en la forma en que se presenta es ambiguo. deben señalarse desde el título las variables o las teorías desde las que se aborda el trabajo.

\section{Resumen}

Es una buena sección pero se puede mejorar tomando en cuenta el estilo de APA para redactar citas y resultados numéricos. Además, los resultados que se muestran en el resumen deben de ser congruentes con los que se muestran en el apartado de resultados como tal. La parte de metodología se puede mejorar tomando en cuenta la descripción de la muestra de una manera más clara y completa. Las palabras clave no reflejan del todo el sentido del artículo, sólo lo haría "rendimiento académico"; las palabras "factores", "actores" y "correlación" son vagas y poco precisas. La palabra clave "obstáculos epistemofílicos" no es parte del estudio como tal, sólo se incluye en un párrafo de las conclusiones, así que no se debería tomar en cuenta.

No menciona cuales son los factores sociopsicopedagogicos favorables, como consecuencia, el propósito del estudio queda ambiguo. En el quinto renglón, la sugerencia es corregir en el nombre del instrumento y que quede con mayúsculas Nivel Superior, dado que es parte del título del instrumento, igual en la cita del autor agregar una coma después del nombre, en el paréntesis. La referencia del autor (Flores, 2012), viene mal citada. Sétimo renglón: quitar un espacio antes de la palabra "muestran" Más adelante, no aclara si los datos de (.042 a .194) son coeficientes de correlación, de ser así, pues no hay relación, se sugiere incluir los niveles de significancia de las correlaciones. Al final de noveno renglón cambiar "tan solo" a "tan sólo". En el décimo renglón, eliminar un espacio después de "por tanto". Igual en el renglón 14, eliminar un espacio antes de la palabra obstáculos. Corregir en el penúltimo renglón: el concepto es epistemológicos no epistemofílicos, y la cita es (Bachelard, 1985) y no (Bachelar, 1985). Al final: cambiar la palabra "keywords" a "palabras clave". Y verificar si realmente los términos incluidos son palabras clave. 


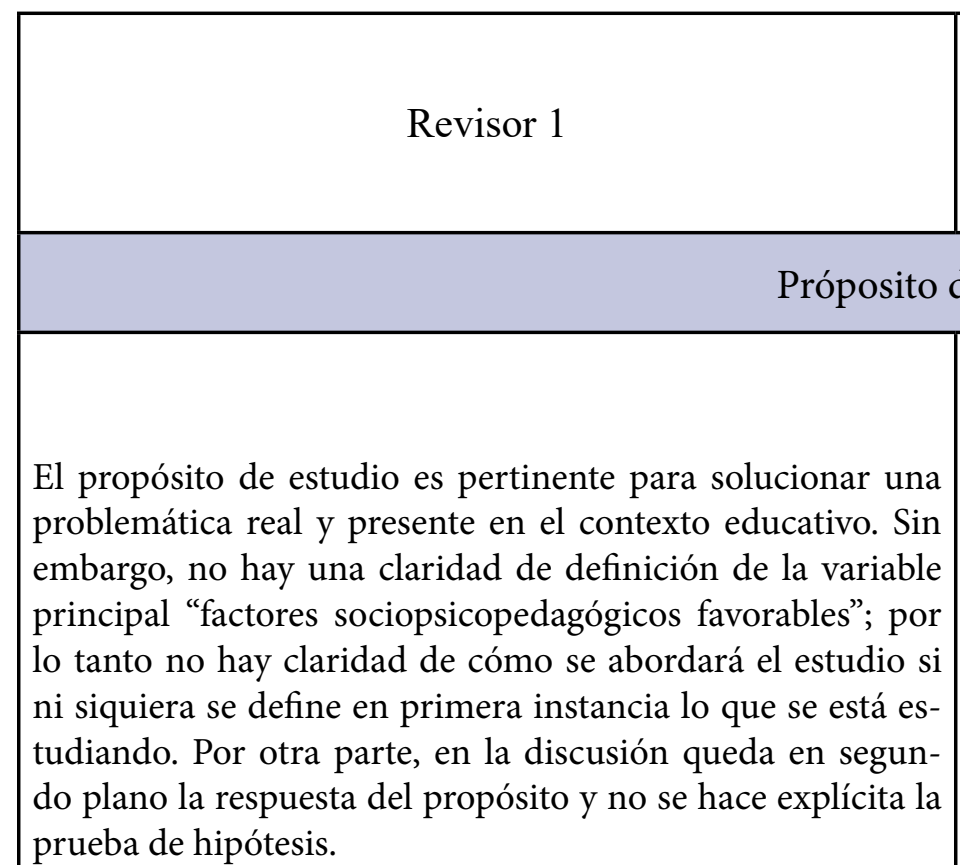

Revisor 2

\section{Introducción}

La introducción posee la mayoría de los elementos necesarios para justificar el propósito de estudio. Sin embargo, la redacción, la ortografía y el citado en APA son deficientes. Para ser considerado un escrito de divulgación científica debe de mejorarse la presentación de ideas, la claridad de exposición y la congruencia entre lo que se mide y lo que se desarrolla en este apartado introductorio. En añadidura Revisar los estudios directamente relacionados con el tema de investigación Redactar la introducción de manera clara y lógica.

El dato de Avilés (2007) es vigente para 2015? Por qué no usar una referencia más reciente y preferentemente que no sea la cita de un periódico, sino de un investigador en educación. Al inicio las ideas no son claras, la redacción no es directa. Al final del segundo párrafo de la página 5 se dice de un "concepto legendario", la idea es que el término sea más técnico y no de leyenda. En la página 6, segundo párrafo, se sugiere incluir una coma después de asimismo, al final colocar el punto final al final y una coma después del autor. En lo que parece ser el tercer párrafo, no queda claro si es un renglón o un título. A lo largo del cuerpo del trabajo, la redacción no es clara. No hay un planteamiento del problema. Hay referencias mal citadas. No hay un planteamiento del problema. pero no menos importante, la definición de factores sociopsicopedagógicos no se identifica, lo cual es muy grave ya que el estudio parte de esta definición.

\section{Método}

La sección es deficiente para la difusión científica del estudio ya que no hay orden de ideas, no se exponen las características de la muestra, ni los detalles del procedimiento, ni hay claridad en la medición realizada. La falta de información sobre el instrumento utilizado es muy grave, ya que en este instrumento se basa todo el estudio y carece desde este punto de validez interna.
Se sugiere incluir objetivo e hipótesis en el método y en el lugar que corresponde. No se aclara si es una o son dos hipótesis. La cita de Creswell (2008), pudiera quedar después de mencionar la muestra. En el segundo párrafo de la página 12, falta un punto final. Instrumento: Corregir la redacción inicial del párrafo. Incluir confiabilidad, validez y descripción del instrumento. Variables: Si es un estudio de tipo correlacional, ¿por qué hay variables dependientes? 


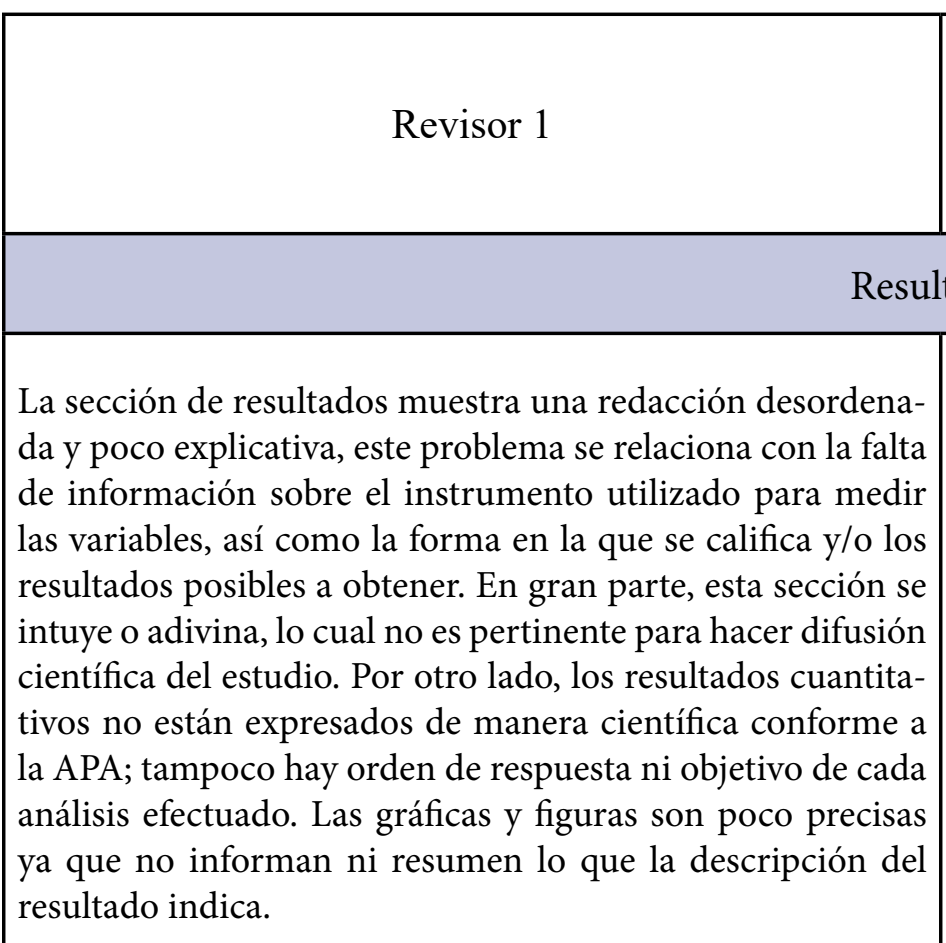

\section{Revisor 2}

La explicación del primer párrafo, no es pertinente. En la columna A mencionan que los participantes no contestaron completamente a las categorías del estudio, pero no mencionan ¿qué estrategias se utilizaron para analizar los datos? No hay columna B, no mencionan por qué la omitieron. Los datos de la columna $\mathrm{F}$, claramente indican que no existe relación entre ninguna de las variables. Pero no mencionan los nieles de significancia asociados. Figura 1 El título no corresponde con la imagen de la gráfica.

\section{Discusión}

Esta sección es redundante con la introducción en la mayoría de su contenido; al discutir los resultados encontrados se hace de manera muy breve y vaga haciendo énfasis en algunos cuantos, discutiendo de manera imprecisa y poco clara los objetivos del estudio y las hipótesis. Las conclusiones a las que se llegan no son lo suficientemente concisas, y en ningún momento se discuten las limitaciones del estudio, El manuscrito no tiene una sección de discusión. lo cual es muy grave, ya que se pretende generalizar los resultados cuando no hay condiciones para afirmarlo. El lado positivo, es que al final del texto se da un giro interesante de información, mostrando que es relevante continuar indagando sobre el tema.

\section{Conclusiones}

La discusión y conclusiones se fusionaron en una sola sección. Mismo comentario anterior.

No se relacionan con la teoría y los hallazgos no tienen base con los resultados- Por cierto, nunca mencionan a Bachelard (1985).

\section{Referencias}

La lista de referencias no tiene uniformidad de formato APA, al igual que sus citas. Además, aproximadamente un $10 \%$ de las referencias no se encuentran citadas en el texto o no hay correspondencia entre lo que se cita y la lista. Esta sección se tiene que trabajar con meticulosidad.

No se apegan al formato APA y con bastantes errores. 
Revista Digital Internacional de Psicología y Ciencia Social |Vol. 2 | Núm. $1 \mid$ Enero-Junio 2016 | ISSSN 1405-5802

Historia del Proceso

\section{EDITORIAL}

